\title{
Monopole and dipole transitions of the cluster states of ${ }^{18} \mathrm{O}$
}

\author{
T. Baba \\ Kitami Institute of Technology, 090-8507 Kitami, Japan \\ M. Kimura \\ Department of Physics, Hokkaido University, 060-0810 Sapporo, Japan \\ Reaction Nuclear Data Centre (JCPRG), \\ Hokkaido University, 060-0810 Sapporo, Japan and \\ Research Center for Nuclear Physics (RCNP), \\ Osaka University, 567-0047 Ibaraki, Japan
}

(Dated: May 11, 2020)

\begin{abstract}
On the basis of an extended antisymmetrized molecular dynamics calculation, we study the cluster structure and the of the $0^{+}$and $1^{-}$states of ${ }^{18} \mathrm{O}$. We discuss that several different kinds of the cluster states appear in the excitation spectrum, and their monopole and dipole transitions are interesting fingerprints of unique cluster structure. We show that the monopole/dipole transitions are enhanced for the ${ }^{14} \mathrm{C}+\alpha$ cluster states, while they are hindered for the molecular-orbit state. We also point out that the ratio of the electric and isoscalar monopole transition strengths gives a good hint for the structure of the excited states.
\end{abstract}




\section{INTRODUCTION}

The nucleus ${ }^{18} \mathrm{O}$ has been an important testing ground for our understanding of the clustering in $N \neq Z$ nuclei. It is of importance and interest to investigate how the extra neutrons affect and enrich the clustering, since the core nucleus ${ }^{16} \mathrm{O}$ has the famous ${ }^{12} \mathrm{C}+\alpha$ cluster states $[1-7]$.

A number of experimental [8 18] and theoretical [19-26] studies have ever explored the ${ }^{14} \mathrm{C}+\alpha$ cluster states in the spectrum of ${ }^{18} \mathrm{O}$. They firmly established the positive-parity band built on the $0_{2}^{+}$state at $3.63 \mathrm{MeV}$, which has a ${ }^{14} \mathrm{C}\left(0_{1}^{+}\right)+\alpha$ cluster structure [8, 19, 20, 23-26]. Due to the parity asymmetry of the ${ }^{14} \mathrm{C}+\alpha$ configuration, this band should be accompanied by the negative-parity band (parity doublet). However, the assignment of the negative-parity band has been rather controversial and unsettled. Gai et al. [9, 10] assigned the $1_{1}^{-}$state at $4.46 \mathrm{MeV}$ as the doublet partner of the $0_{2}^{+}$state based on the enhanced $E 1$ transition strength between them. However, this assignment was not supported by the theoretical calculations [23 26]. For example, from the multi-configuration cluster model calculations, Descouvemont and Baye [23] pointed out that the $4.46 \mathrm{MeV}$ state is predominated by the ${ }^{14} \mathrm{C}\left(2^{+}\right)+\alpha$ channel, and hence, cannot be considered as the partner of the $0_{2}^{+}$state. Alternatively, they showed that the calculated $1_{3}^{-}$state has the pronounced ${ }^{14} \mathrm{C}\left(0_{1}^{+}\right)+\alpha$ cluster structure, and tentatively assigned it to the $1^{-}$state observed at 7.62

$\mathrm{MeV}$ [8, 27]. Later, this assignment was corrected by several experiments [13, 14] which assigned a new negative-parity band built on the $1^{-}$state at $8.03 \mathrm{MeV}$ as the partner of the $0_{2}^{+}$state. A confusing fact is that this assignment was again denied by another recent experiment: Avila et al. [17] reported that the $\alpha$ spectroscopic factor of the $8.03 \mathrm{MeV}$ state is not large, and hence, the state cannot be a ${ }^{14} \mathrm{C}\left(0_{1}^{+}\right)+\alpha$ cluster state.

In addition to the ${ }^{14} \mathrm{C}+\alpha$ cluster states, von Oertzen et al. [16] proposed a novel type of cluster states which are composed of the ${ }^{12} \mathrm{C}+\alpha$ cluster core and two valence neutrons occupying so-called molecular orbits (MO state). They tentatively assigned the 7.80 and 10.59 $\mathrm{MeV}$ states as the $0^{+}$and $1^{-}$doublet of the MO states. The existence of such MO state was qualitatively supported by the antisymmetrized molecular dynamics (AMD) calculations [26], and experimental efforts to find more convincing evidence is now on going [18]. Thus, identifying the pair of the $0^{+}$and $1^{-}$cluster states is important to understand a rich variety of clustering systematics in ${ }^{18} \mathrm{O}$. 
In this decade, the isoscalar monopole and dipole transition strengths are attracting a lot of interest as a novel probe for the $0^{+}$and $1^{-}$cluster states, and have already been used for the discussion of the clustering in many stable and unstable nuclei [28-45]. Therefore, we expect that they provide a new insight to the clustering of ${ }^{18} \mathrm{O}$.

For this purpose, we perform an extended AMD calculation for ${ }^{18} \mathrm{O}$ taking into account the coupling of the ${ }^{14} \mathrm{C}\left(0_{1}^{+}\right)+\alpha$ and ${ }^{14} \mathrm{C}\left(2_{1}^{+}\right)+\alpha$ channels. We analyze the cluster structure of the $0^{+}$and $1^{-}$states referring their $\alpha$-spectroscopic factors, and investigate how the clustering affects the monopole and dipole transition strengths. We show that the dipole transition strength between the doublet of the ${ }^{14} \mathrm{C}+\alpha$ cluster states is greatly enhanced, while that of the MO state is hindered. We also discuss that the ratio of the electric and isoscalar monopole transition strengths also gives us an interesting hints on the cluster structure.

This paper is organized as follows: In the next section, we briefly explain how we calculated the wave functions of the cluster states of ${ }^{18} \mathrm{O}$. We also explain the electric and isoscalar monopole/dipole transition matrices. In the section III, we first review the calculated and observed spectrum of the cluster states. And we investigate how the characteristics of each cluster states is reflected to the pattern of the transition strengths. The final section summarizes this work.

\section{THEORETICAL FRAMEWORK}

In this study, we use the Hamiltonian same with our previous study [26],

$$
H=\sum_{i=1}^{A} t_{i}-t_{\text {c.m. }}+\sum_{i<j}^{A} v_{i j},
$$

where $t_{i}$ and $t_{\text {c.m. }}$ represents the single-particle and center-of-mass kinetic energies. $v_{i j}$ includes the Gogny D1S effective nucleon-nucleon interaction [46] and Coulomb interaction.

The model wave function is a parity-projected Slater determinant,

$$
\Phi_{\mathrm{AMD}}=P^{\pi} \mathcal{A}\left\{\varphi_{1}, \ldots, \varphi_{A}\right\}, \quad \pi= \pm,
$$

where $P^{\pi}$ is the parity-projection operator, and the single-particle wave packets has the 
deformed Gaussian form [47-49],

$$
\begin{aligned}
\varphi_{i}(\boldsymbol{r})= & \prod_{\sigma=x, y, z} \exp \left\{-\nu_{\sigma}\left(r_{\sigma}-Z_{i \sigma}\right)^{2}\right\} \\
& \otimes\left(\alpha_{i}|\uparrow\rangle+\beta_{i}|\downarrow\rangle\right) \otimes(|p\rangle \text { or }|n\rangle) .
\end{aligned}
$$

Each Gaussian wave packet has the variational parameters: The Gaussian centroid vector $\boldsymbol{Z}_{i}$ and the spin parameters $\alpha_{i}$ and $\beta_{i}$. The isospin is fixed to either of proton or neutron. The Gaussian width parameters $\nu_{x}, \nu_{y}$ and $\nu_{z}$ are also the variational parameters and common to all wave packets.

These variational parameters are determined by the following two methods. The first is the energy variation with the constraint, which was already used in our previous study [26]. Using the frictional cooling method, the variational parameters are so chosen to minimize the total energy under the constraint on the quadruple deformation parameter $\beta$. By this calculation, we obtain the optimized wave function $\Phi_{\mathrm{AMD}}(\beta)$ for each value of $\beta$ ( $\beta=$ $0.00,0.05, \ldots, 1.40)$. As discussed in Ref. [26], if $\beta$ is small, we obtain the almost spherical shell-model-like wave functions corresponding to the ground state, but as $\beta$ increases, we obtain a variety of cluster configurations such as the ${ }^{14} \mathrm{C}+\alpha$ cluster, molecular-orbit state and linear-chain of $\alpha$ particles.

In this study, we extend the model space by applying the second method. We use the Brink-type wave function for ${ }^{14} \mathrm{C}+\alpha$ configurations, in which ${ }^{14} \mathrm{C}$ and $\alpha$ cluster wave functions are placed on the $z$-axis with the inter-cluster distance $d$,

$$
\Phi_{\text {Brink }}(d)=P^{\pi} \mathcal{A}\left\{\Phi_{\alpha}\left(-\frac{14}{18} d\right) \Phi_{\mathrm{C}}\left(\frac{4}{18} d\right)\right\}
$$

where $\Phi_{\alpha}$ and $\Phi_{\mathrm{C}}$ represents the intrinsic wave functions of ${ }^{4} \mathrm{He}$ and ${ }^{14} \mathrm{C}$, respectively. $\Phi_{\alpha}$ is assumed to have the $(0 s)^{4}$ configuration, and $\Phi_{\mathrm{C}}$ is approximated by a single AMD wave function which has 90\% overlap with the full GCM wave function obtained in Ref. [50, 51]. The inter-cluster distance $d$ ranges from $0.4 \mathrm{fm}$ to $8.0 \mathrm{fm}$ with the intervals of $0.4 \mathrm{fm}$. Since $\Phi_{\mathrm{C}}$ is oblately deformed, we consider three different orientations of ${ }^{14} \mathrm{C}$ cluster. Figure 1 shows the example of the ${ }^{14} \mathrm{C}+\alpha$ Brink wave functions in which the symmetry axis of ${ }^{14} \mathrm{C}$ is directed to the (a) $z$-, (b) $x$-, and (c) $y$-axis. Note that the superposition of different orientations of $\Phi_{\mathrm{C}}$ naturally handles the coupling of the ${ }^{14} \mathrm{C}\left(0_{1}^{+}\right)+\alpha$ and ${ }^{14} \mathrm{C}\left(2_{1}^{+}\right)+\alpha$ channels, which is known to be important in describing the ${ }^{14} \mathrm{C}+\alpha$ clustering [23]. This point is an advantage of the present calculation compared to previous AMD studies. 


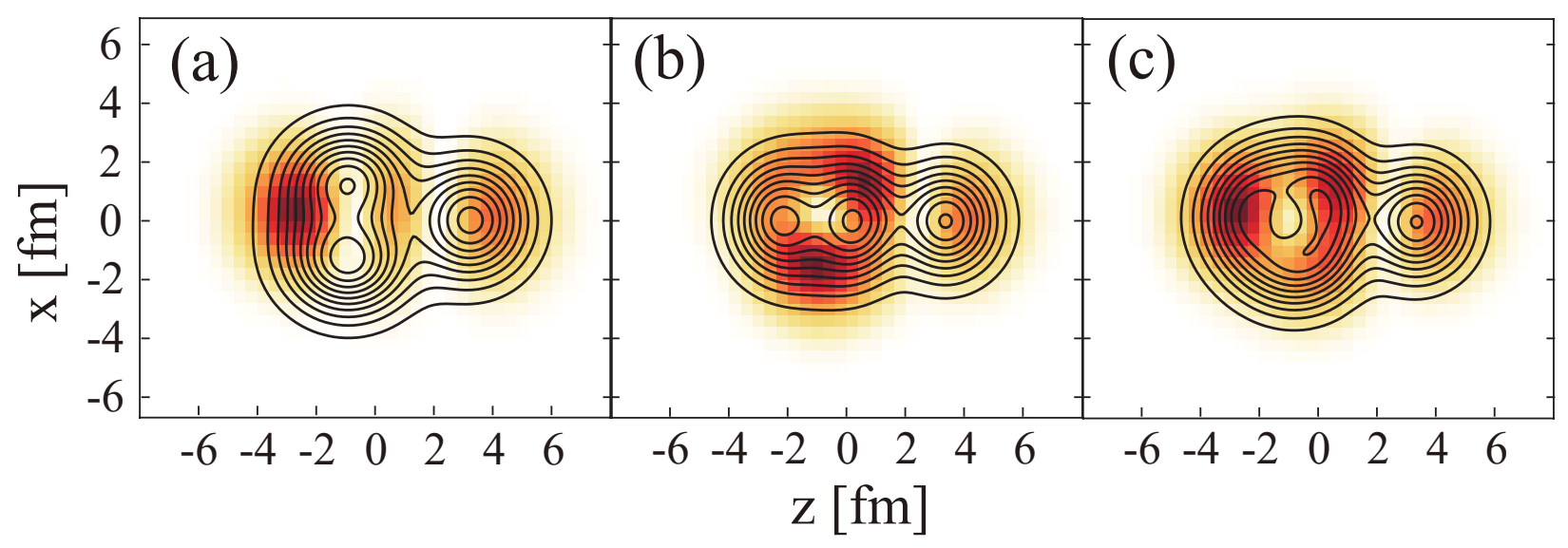

FIG. 1. (color online) Density distributions of the Brink-type wave functions in which the symmetry axis of the oblately deformed ${ }^{14} \mathrm{C}$ is directed to the (a) $z-$, (b) $x$-, and (c) $y$-axis. The inter-cluster distance $d$ is fixed to $4.8 \mathrm{fm}$ in all panels.

These wave functions are projected to the eigenstate of the angular momentum and are superposed to describe the ground and excited states,

$$
\begin{aligned}
\Psi_{M p}^{J^{\pi}}= & \sum_{K i} f_{K i p} P_{M K}^{J} \Phi_{\mathrm{AMD}}^{\pi}\left(\beta_{i}\right) \\
& +\sum_{K i} g_{K i p} P_{M K}^{J} \Phi_{\mathrm{Brink}}^{\pi}\left(d_{i}\right),
\end{aligned}
$$

where $P_{M K}^{J}$ and the index $p$ denote the angular momentum projection operator and the quantum number other than the angular momentum, respectively. The coefficients $f_{\text {Kip }}$ and $g_{K i p}$ are determined by diagonalizing Hamiltonian [52].

As a measure of the ${ }^{14} \mathrm{C}+\alpha$ clustering, we calculate the $\alpha$-spectroscopic factor. We first calculate the $\alpha$ reduced width amplitudes (RWA) which is the probability amplitude to find the ${ }^{14} \mathrm{C}$ and $\alpha$ clusters at the inter-cluster distance $a$. It is defined as the overlap between the reference cluster state and the wave function given by Eq. (5),

$$
\begin{aligned}
y_{j \ell J}(a) & =\sqrt{\frac{18 !}{14 ! 4 !}}\left\langle\frac{\delta(r-a)}{r^{2}} \Phi_{\alpha}\left[\Phi_{\mathrm{C}}^{j} Y_{\ell}(\hat{r})\right]_{M}^{J} \mid \Psi_{M p}^{J \pi}\right\rangle, \\
(j & \left.=0^{+} \text {or } 2^{+}\right) .
\end{aligned}
$$

The reference cluster state (bra state) is the ${ }^{14} \mathrm{C}(j)+\alpha$ cluster state in which the ${ }^{14} \mathrm{C}(j)$ and $\alpha$ clusters are mutually orbiting with inter-cluster distance $a$, and the intrinsic angular momentum $j$ of the ${ }^{14} \mathrm{C}$ cluster is coupled with the orbital angular momentum $\ell$ to the total 
angular momentum $J$. Here, the $\alpha$ cluster wave function $\Phi_{\alpha}$ is same with that appears in Eq. (4), and the ${ }^{14} \mathrm{C}$ cluster wave function $\Phi_{\mathrm{C}}^{j}$ also uses the intrinsic wave function $\Phi_{\mathrm{C}}$ same with that appears in Eq. (4) but projected to the eigenstate of the angular momentum $j^{\pi}=0^{+}$or $2^{+}$. In the practical calculation, Eq. (6) is evaluated by the Laplace expansion method [53]. The $\alpha$ spectroscopic factor $S_{\alpha}$ is given by a squared integral of $y_{j \ell J}$.

$$
S_{\alpha}=\int_{0}^{\infty} d a a^{2}\left|y_{j \ell J}(a)\right|^{2}
$$

In this work, we focus on the electric and isoscalar monopole ( $E 0$ and $I S 0)$ and dipole ( $E 1$ and $I S 1$ ) transition strengths. As discussed in Refs. [9, 10], the $E 1$ transition strength is a good probe for the ${ }^{14} \mathrm{C}+\alpha$ cluster states, since the intrinsic structure has static dipole moment. In addition to the $E 1$ transition strength, in this decade, the E0, ISO and IS 1 transition strengths have been regarded and utilized as a novel probe for the various kinds of clustering [31, 39], as it was proved that these transitions from the ground state to an excited cluster state must be considerably enhanced. The transition operators are defined as follows,

$$
\begin{aligned}
& \mathcal{M}^{E 0}=\sum_{i=1}^{Z} e r_{i}^{\prime 2}, \quad \mathcal{M}^{I S 0}=\sum_{i=1}^{A} r_{i}^{\prime 2}, \\
& \mathcal{M}_{\mu}^{E 1}=\sum_{i=1}^{Z} e r_{i}^{\prime} Y_{1 \mu}\left(\hat{r}_{i}^{\prime}\right), \quad \mathcal{M}_{\mu}^{I S 1}=\sum_{i=1}^{A} r_{i}^{\prime 3} Y_{1 \mu}\left(\hat{r}_{i}^{\prime}\right) .
\end{aligned}
$$

Note that the single-particle coordinate $\boldsymbol{r}_{i}^{\prime}$ is measured from the center-of-mass $\boldsymbol{r}_{\text {c.m. }}$, i.e. $\boldsymbol{r}_{i}^{\prime} \equiv$ $\boldsymbol{r}_{i}-\boldsymbol{r}_{\text {c.m. }}$, and hence, our calculation is free from the spurious center-of-mass contributions. The transition strength from the initial state $\Psi_{0 p}^{J^{\pi}}$ to the $0^{+}$state is evaluated by the reduced transition matrix,

$$
M\left(\lambda ; J^{\pi} \rightarrow 0^{+}\right)=\left\langle\Psi^{0^{+}}\left|\mathcal{M}_{0}^{\lambda}\right| \Psi_{0 p}^{J^{\pi}}\right\rangle
$$

where $\mathcal{M}_{0}^{\lambda}$ is any of the transition operators where $\lambda$ is either of $E 0, I S 0, E 1$ and $I S 1$.

\section{RESULTS}

\section{A. Cluster states and their structure}

Figure 2 shows the spectrum of the $0^{+}$and $1^{-}$states obtained by the present calculation

compared with the other theoretical calculations [23, 25]. It also shows the proposed assignments of the cluster states based on the experiments [8, 10, 13, 14, 16, 17, 27]. In the present 


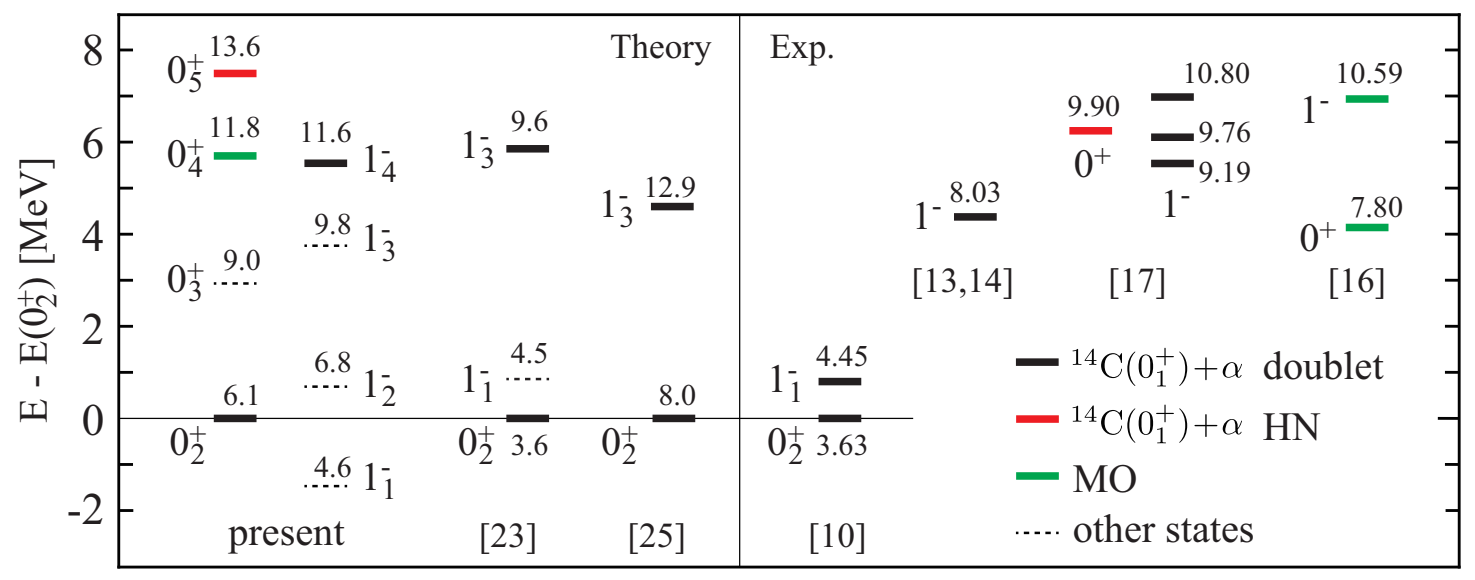

FIG. 2. (color online) Spectrum of the $0^{+}$and $1^{-}$states obtained by the present calculation in comparison with other model calculations [23, 25]. Vertical axis shows the energy relative to the $0_{2}^{+}$state, while numbers in the figure show the energies relative to the ground state. The proposed assignments of the cluster states $1^{-}\left(0^{+}\right)$with cluster configurations based on the experiments [8, 10, 13, 14, 16, 17, 27] are also shown.

calculation, the bandhead of the positive-parity ${ }^{14} \mathrm{C}\left(0_{1}^{+}\right)+\alpha$ cluster band is obtained as the $0_{2}^{+}$state at $6.1 \mathrm{MeV}$ which slightly overestimates the observed excitation energy $(3.63 \mathrm{MeV})$. From the calculated spectroscopic factor shown in Fig. 3, this assignment is rather unique as only this state is dominated by the ${ }^{14} \mathrm{C}\left(0_{1}^{+}\right)+\alpha$ channel in the low-energy region. The intrinsic density of this state shown in Fig. 4 (b) also shows a moderate ${ }^{14} \mathrm{C}+\alpha$ clustering which is clearly different from the ground state (Fig $4(\mathrm{a})$ ).

The corresponding negative-parity partner is also uniquely identified as the $1_{4}^{-}$state at 11.6 MeV. It is clear that other $1^{-}$states have relatively small spectroscopic factors and are excluded from the doublet partner. For example, the intrinsic density of the $1_{1}^{-}$state (Fig. 4 (e)) clearly shows the absence of the prominent $\alpha$ clustering in this state. Thus, the present calculation yields $5.5 \mathrm{MeV}$ energy splitting between the $0^{+}$and $1^{-}$doublet of the ${ }^{14} \mathrm{C}\left(0_{1}^{+}\right)+\alpha$ configuration, which is larger than that of the ${ }^{12} \mathrm{C}+\alpha$ doublet of ${ }^{16} \mathrm{O}(3.5$ $\mathrm{MeV})$, and as large as that of the ${ }^{16} \mathrm{O}+\alpha$ doublet of ${ }^{20} \mathrm{Ne}(5.9 \mathrm{MeV})$. This indicates the distortion of the ${ }^{14} \mathrm{C}\left(0_{1}^{+}\right)+\alpha$ clustering in the positive parity state, which brings about extra binding energy to the $0^{+}$state and enlarges the doublet splitting. It is notable that other theoretical calculations also yielded similar magnitudes of the splitting: Furutachi et al. [25] reported the $4.9 \mathrm{MeV}$ splitting from their AMD calculation which used a different 
effective interaction and model wave function from ours. Descouvemont et al. [23] reported 6.0 MeV splitting from their multi-configuration cluster model calculation. Thus, theoretical calculations suggest the consistent magnitudes of the doublet splitting approximately equal to $5 \sim 6 \mathrm{MeV}$.
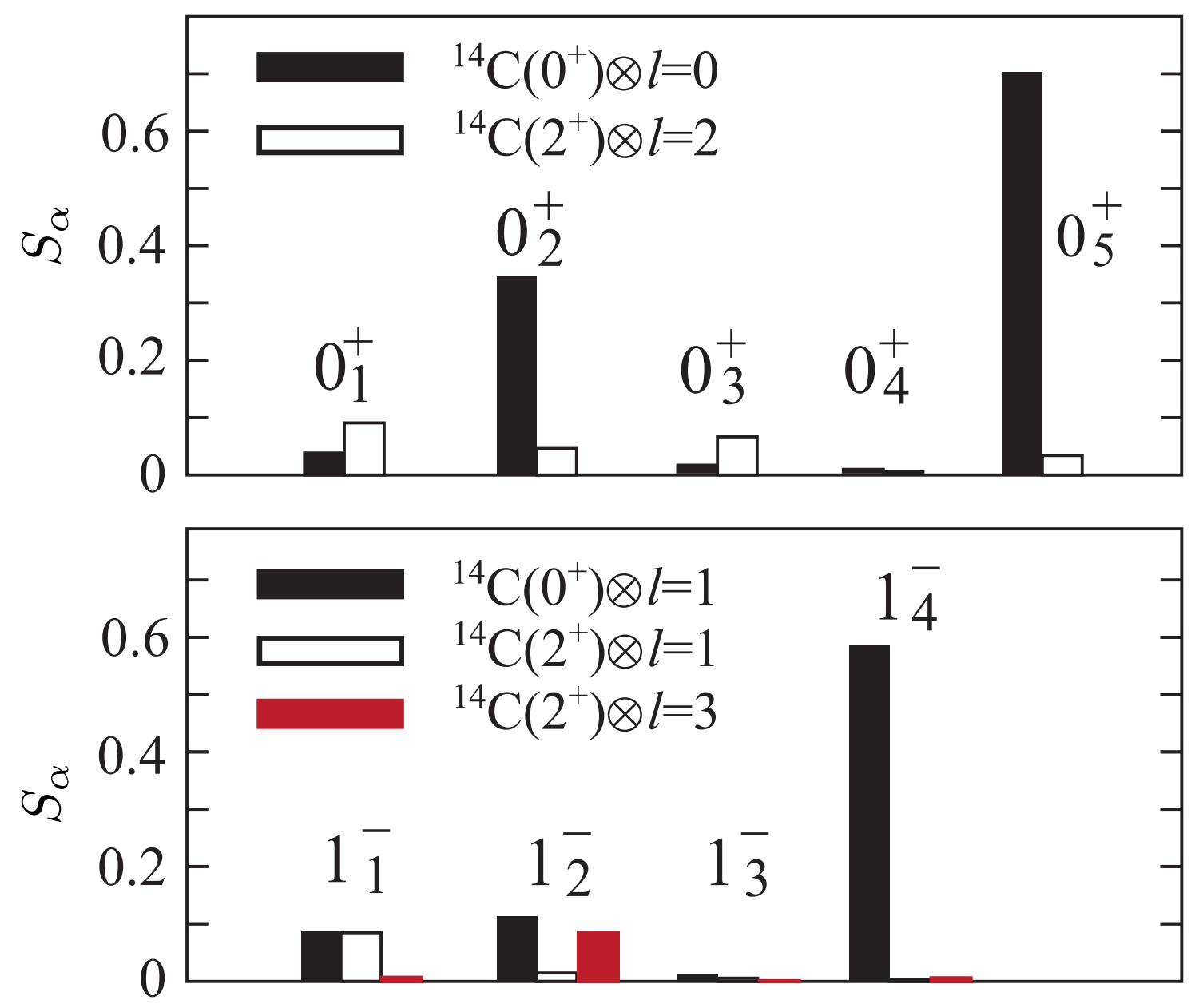

FIG. 3. (color online) Calculated $\alpha$ spectroscopic factors of the $0^{+}$and $1^{-}$states.

Experimentally, the assignment of the positive-parity ${ }^{14} \mathrm{C}\left(0_{1}^{+}\right)+\alpha$ state is well established and unique [8, 19, 20, 23 26]; it is the $0_{2}^{+}$state at $3.63 \mathrm{MeV}$, but the assignment of the negative-parity state is controversial. Gai et al. [10] proposed the $1_{1}^{-}$state at $4.45 \mathrm{MeV}$ as the partner of the $0_{2}^{+}$state, because the ${ }^{14} \mathrm{C}+\alpha$ intrinsic structure can naturally explain the observed strong $E 1$ transition between them. However, this assignment gives very small doublet splitting $0.82 \mathrm{MeV}$ which contradicts to all theoretical calculations. Furthermore, the present calculation shows the $1_{1}^{-}$state is a mixture of the small amount of the ${ }^{14} \mathrm{C}\left(0_{1}^{+}\right)+\alpha$ and ${ }^{14} \mathrm{C}\left(2_{1}^{+}\right)+\alpha$ components, which is consistent with the results by Descouvemont et al. 


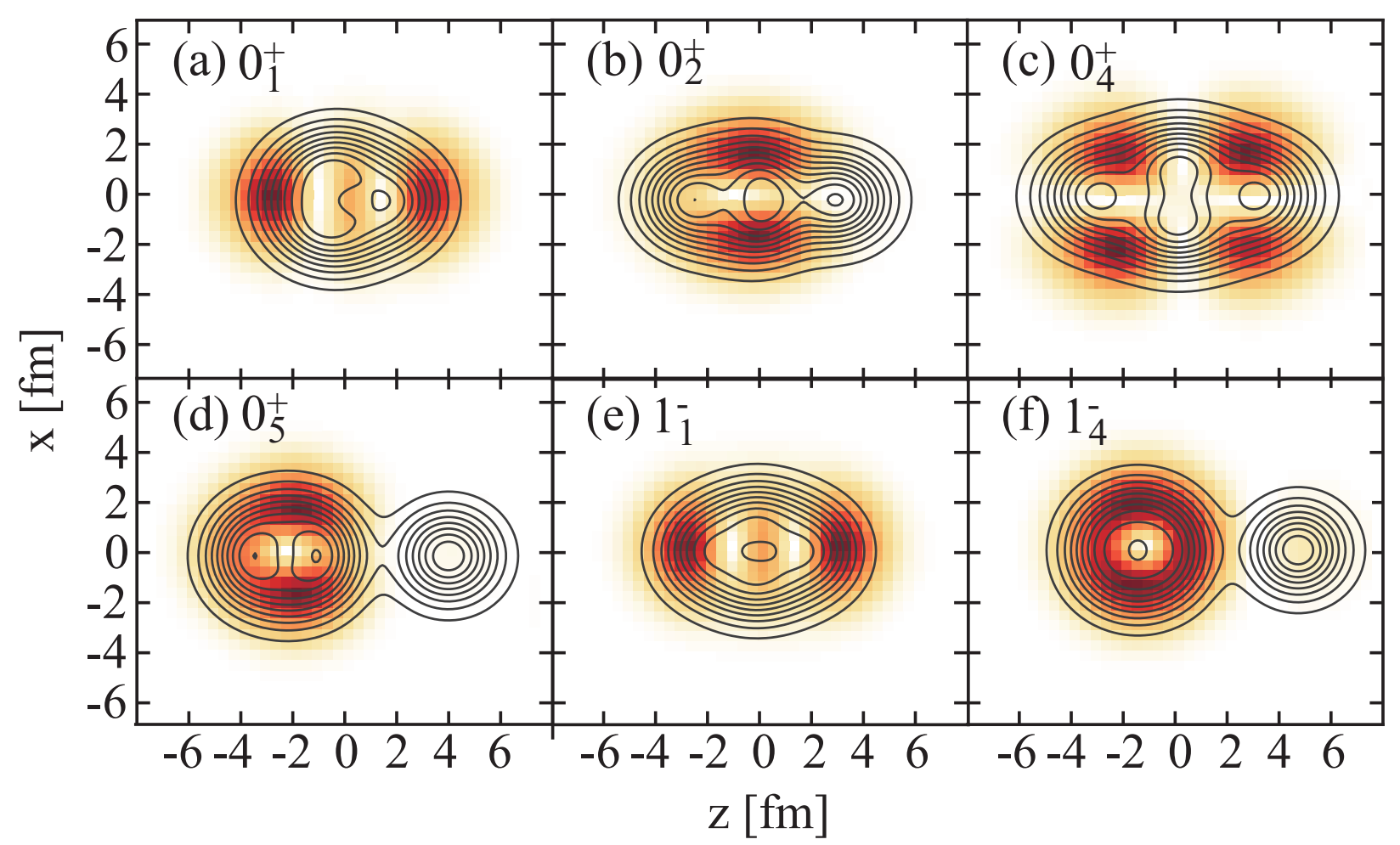

FIG. 4. (color online) Density distributions of the intrinsic states which are the dominant component of each $0^{+}$or $1^{-}$state. Solid lines show the proton density distribution, while the color plot show that of of two valence neutrons.

[23], but contradict to the assignment by Gai et al. Other candidates of the doublet partner are the the $1^{-}$state at $8.03 \mathrm{MeV}$ observed by the breakup reaction [13, 14] and the 9.19, 9.76 and $10.39 \mathrm{MeV}$ states observed by the resonant scattering [17]. In these assignments, the magnitudes of the doublet splitting are approximately in between 4 to $7 \mathrm{MeV}$. Thus, all these assignments look compatible with the theoretical results, but there is no conclusive evidence.

In addition to the ${ }^{14} \mathrm{C}+\alpha$ doublet, the present calculation predicts two excited cluster states; the $0^{+}$states at $11.8 \mathrm{MeV}\left(0_{4}^{+}\right)$and $13.6 \mathrm{MeV}\left(0_{5}^{+}\right)$whose intrinsic densities are shown in Fig. 4 (c) and (d), respectively. The $11.8 \mathrm{MeV}$ state has the ${ }^{12} \mathrm{C}+\alpha$ cluster core surrounded by the valence neutrons occupying so-called molecular orbit (MO state), which is similar to those known for Be, $\mathrm{C}$ and Ne isotopes [50, 54 58]. We consider that this state may correspond to the MO structure suggested by von Oertzen who proposed a tentative assignment to the $7.80 \mathrm{MeV}$ state [16]. The $0_{5}^{+}$state at $13.6 \mathrm{MeV}$ is the ${ }^{14} \mathrm{C}+\alpha$ higher-nodal state (HN state), in which the relative motion between the ${ }^{14} \mathrm{C}$ and $\alpha$ clusters is excited as 
seen in its density distribution (Fig. 4 (d)). The corresponding observed state might be the $9.90 \mathrm{MeV}$ state reported by Avila et al. [17], as it is the only $0^{+}$state which has large spectroscopic factor in this energy region.

In short, theoretical calculations predict the doublet of the $0^{+}$and $1^{-}$states with the ${ }^{14} \mathrm{C}+\alpha$ configuration, but the assignment of the $1^{-}$state has not been established uniquely. The ${ }^{14} \mathrm{C}+\alpha \mathrm{HN}$ and MO states are also suggested by the experiments and the present calculation.

\section{B. Monopole and dipole transitions to and between the cluster states}

Here, we investigate how the characteristics of the cluster states discussed in the previous section are reflected to the $E 0, I S 0, E 1$ and $I S 1$ transition strengths listed in Tab. I. For this purpose, the pattern of the calculated transitions is schematically illustrated in Fig. 5 .

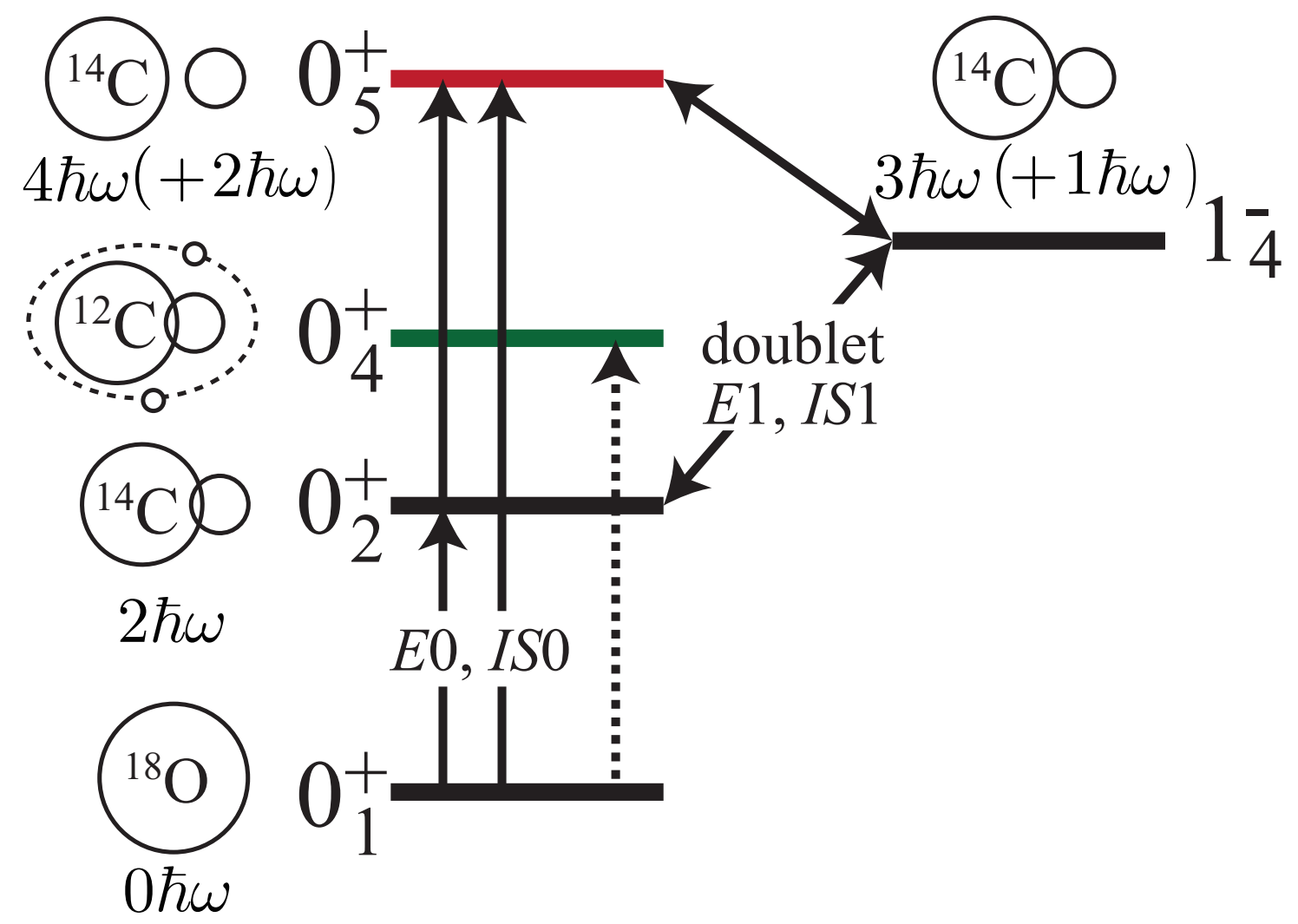

FIG. 5. (color online) Schematic figure which illustrates the cluster states of ${ }^{18} \mathrm{O}$ and the pattern of the transitions among them.

In Ref. [31], Yamada al. proved that the electric and isoscalar monopole transitions from 
TABLE I. The calculated reduced matrix for the E0, ISO,E1 and $I S 1$ transitions in Weisskopf unit (in $10^{-2}$ W.u. for the $E 1$ transitions). 1 W.u. is equal to $5.93 \mathrm{efm}^{2}, 5.93 \mathrm{fm}^{2}, 0.665 \mathrm{efm}$ and $4.39 \mathrm{fm}^{3}$ for the $E 0, I S 0, E 1$ and $I S 1$ transitions, respectively.

\begin{tabular}{lcc}
\hline \hline$J_{i}^{\pi} \rightarrow J_{f}^{\pi}$ & $M^{E 0}[$ W.u. $]$ & $M^{I S 0}[\mathrm{~W} . \mathrm{u}]$. \\
\hline $0_{1}^{+} \rightarrow 0_{2}^{+}$ & 0.36 & 0.67 \\
$0_{1}^{+} \rightarrow 0_{3}^{+}$ & 0.28 & 0.83 \\
$0_{1}^{+} \rightarrow 0_{4}^{+}$ & 0.02 & 0.02 \\
$0_{1}^{+} \rightarrow 0_{5}^{+}$ & 0.28 & 0.56 \\
$0_{2}^{+} \rightarrow 0_{5}^{+}$ & 1.31 & 2.75 \\
& $M^{E 1}\left[10^{-2} \mathrm{~W} . \mathrm{u.}\right]$ & $M^{I S 1}[\mathrm{~W} . \mathrm{u.}]$ \\
\hline $1_{1}^{-} \rightarrow 0_{1}^{+}$ & 1.20 & 0.77 \\
$1_{2}^{-} \rightarrow 0_{1}^{+}$ & 2.53 & 0.51 \\
$1_{3}^{-} \rightarrow 0_{1}^{+}$ & 7.99 & 0.40 \\
$1_{4}^{-} \rightarrow 0_{1}^{+}$ & 3.57 & 0.70 \\
$1_{1}^{-} \rightarrow 0_{2}^{+}$ & 5.11 & 1.58 \\
$1_{2}^{-} \rightarrow 0_{2}^{+}$ & 7.23 & 1.74 \\
$1_{3}^{-} \rightarrow 0_{2}^{+}$ & 2.94 & 0.75 \\
$1_{4}^{-} \rightarrow 0_{2}^{+}$ & 17.3 & 5.19 \\
$1_{4}^{-} \rightarrow 0_{5}^{+}$ & 38.7 & 17.4 \\
\hline \hline
\end{tabular}

the ground state to the $\alpha$-cluster state can be considerably enhanced. Since the $\alpha$-cluster states appear at relatively small excitation energy compared to other collective states [59], the strong $E 0$ and $I S 0$ strengths at small excitation energy can be attributed to the $\alpha$-cluster formation. Therefore, the strong monopole transition has been regarded as a signature of the cluster states in stable and unstable nuclei [28 38, 41, 44, 45]. In the present calculation, as expected, we find that the $0_{2}^{+}$state which has a ${ }^{14} \mathrm{C}+\alpha$ cluster structure has non small $E 0$ and $I S 0$ transition strengths from the ground state. However, they are not as strong as the Weisskopf unit because of the following reason. As seen in Fig. 3 and also discussed in Ref. [23], the dominant cluster component of the ground state is the ${ }^{14} \mathrm{C}\left(2_{1}^{+}\right)+\alpha$ channel, 
while that of the $0_{2}^{+}$state is different, the ${ }^{14} \mathrm{C}\left(0_{1}^{+}\right)+\alpha$ channel. This mismatch of the internal structure reduces the monopole strengths between them. The same argument also applies to the $0_{5}^{+}$state. As the $0_{5}^{+}$state is also dominated by the ${ }^{14} \mathrm{C}\left(0_{1}^{+}\right)+\alpha$ channel, the monopole transition from the ground state is not so enhanced. On the contrary, the transition between the $0_{2}^{+}$and $0_{5}^{+}$states is very strong because they have similar internal structure. It is interesting to note that the $I S 0$ matrices for the $0_{1}^{+} \rightarrow 0_{2}^{+}$and $0_{1}^{+} \rightarrow 0_{5}^{+}$ transitions are almost twice as large as the E0 matrices. This is naturally understood as these excitation are $\alpha$ clustering, and hence, proton and neutron should equally contribute to the transitions. On the other hand, for the $0_{1}^{+} \rightarrow 0_{3}^{+}$transition, the $I S 0$ matrix is much larger than twice of the $E 0$ matrix. This is due to the fact that the $0_{3}^{+}$state is not a $\alpha$-cluster state but an excited state predominated by the excitation of valence neutrons. Thus, not only the magnitude but also the ratio of the electric and isoscalar monopole matrices gives us an insight to nuclear structure. We also note that the $0_{1}^{+} \rightarrow 0_{4}^{+}$transitions is rather hindered in both of the electric and isoscalar channels compared to other states, because the $0_{4}^{+}$has a MO structure, and hence, the $0_{1}^{+} \rightarrow 0_{4}^{+}$transition involves the rearrangement of two valence neutrons. A similar hindrance of the monopole transitions was also discussed in Be isotopes [32, 36].

Experimentally, two different values of $M(E 0)$ for the $0_{1}^{+} \rightarrow 0_{2}^{+}$transition were reported: The lower value $0.49 \mathrm{~W} . \mathrm{u}$. is not far from our result, but the larger one $1.01 \mathrm{~W} . \mathrm{u}$. is much larger than ours. There is a possibility that we underestimated the $\alpha$ clustering of the $0_{2}^{+}$ state. In particular, the amount of the ${ }^{14} \mathrm{C}\left(2^{+}\right)+\alpha$ component in the $0_{2}^{+}$state may not be large enough in our calculation, as the transition strength is sensitive to it. We also mention the importance of the rotation effect of ${ }^{14} \mathrm{C}$ cluster. If we calculate the $0_{1}^{+} \rightarrow 0_{2}^{+}$transition without ${ }^{14} \mathrm{C}+\alpha$ Brink-basis wave function, the electric monopole transition strength is 0.18 W.u. which is much smaller than both of the experimental data. As for the $0_{1}^{+} \rightarrow 0_{4,5}^{+}$ transitions, no corresponding experimental data has been reported so far. The measurement of the transition strengths from the ground states to the 7.80 and $9.90 \mathrm{MeV}$ states will provide an interesting hint about the clustering in ${ }^{18} \mathrm{O}$, as they are the candidates of the calculated $0_{4,5}^{+}$states.

The electric and isoscalar dipole transitions are good probe to identify the cluster $1^{-}$ states as they are enhanced between the doublet $\left(0^{+}\right.$and $1^{-}$states $)$[9, 39]. Indeed, the present results confirm that both of the electric and isoscalar dipole transition matrices are 
large for the $1_{4}^{-} \rightarrow 0_{2}^{+}$transition. Furthermore, we found that they are also enhanced for the $1_{4}^{-} \rightarrow 0_{5}^{+}$transition, because of the well developed cluster structure of the $0_{5}^{+}$state. Experimentally, the 8.03 9.19, 9.76 and $10.39 \mathrm{MeV}$ states are the candidates of the $1_{4}^{-}$state which constitutes the doublet with the $0_{2}^{+}$state, but the convincing evidence is missing. Therefore, the information about the magnitude of the dipole transitions of these candidates will be very useful to identify the doublet.

Compared to the $1_{4}^{-} \rightarrow 0_{2}^{+}$transitions, the the $1_{4}^{-} \rightarrow 0_{1}^{+}$transitions are not so enhanced. This may be again due to the mismatch of the internal structure. The ground state is dominated by the ${ }^{14} \mathrm{C}\left(2_{1}^{+}\right)+\alpha$ channel, while the $1_{4}^{-}$state is not but by the ${ }^{14} \mathrm{C}\left(0_{1}^{+}\right)+\alpha$ channel. We note that a similar discussion was also made for the $1_{2}^{-} \rightarrow 0_{1}^{+}$transition of ${ }^{16} \mathrm{O}$ [43]. Finally, we mention the $1_{1}^{-} \rightarrow 0_{2}^{+}$transitions for which a very strong $E 1$ transition was reported by Gai et al. In our calculation, like other theoretical calculations, the enhanced $E 1$ transition was not reproduced, because the $1_{1}^{-}$state exhibits no clustering as seen in its density plot (Fig. 4(e)). This may indicate that some cluster correlations is missing in theoretical calculations. Thus, unfortunately, the inconsistency between the theory and experiment for the $1_{1}^{-}$state was not resolved.

\section{SUMMARY}

To understand the clustering systematics in a $N \neq Z$ nucleus ${ }^{18} \mathrm{O}$, we performed an extended AMD calculation taking into account the coupling of the ${ }^{14} \mathrm{C}\left(0_{1}^{+}\right)+\alpha$ and ${ }^{14} \mathrm{C}\left(2_{1}^{+}\right)+$ $\alpha$ channels, and analyzed the cluster structure of the $0^{+}$and $1^{-}$states. We also investigated to what extent the characteristics of the cluster states are reflected to the pattern of the monopole and dipole transition strengths.

Based on the calculated $\alpha$ spectroscopic factors, we identified the $0_{2}^{+}$and $1_{4}^{-}$states as a doublet of the ${ }^{14} \mathrm{C}\left(0_{1}^{+}\right)+\alpha$ cluster states. This assignment gives $5.5 \mathrm{MeV}$ for the doublet splitting which is consistent with other theoretical calculations. Furthermore, our calculation predicts the $0_{4}^{+}$and $0_{5}^{+}$states, which respectively have the MO structure and the ${ }^{14} \mathrm{C}\left(0_{1}^{+}\right)+\alpha$ HN structure.

From the analysis of the electric and isoscalar monopole transitions, we found that the transitions to the ${ }^{14} \mathrm{C}+\alpha$ cluster states, namely the $0_{1}^{+} \rightarrow 0_{2}^{+}$and $0_{1}^{+} \rightarrow 0_{5}^{+}$transitions, are much stronger than the transition to the MO state $\left(0_{1}^{+} \rightarrow 0_{4}^{+}\right)$. This is a good measure 
to distinguish the ${ }^{14} \mathrm{C}+\alpha$ cluster states and $\mathrm{MO}$ states, and to identify the experimental counterpart of the $0_{5}^{+}$state. We also found that for the $0_{1}^{+} \rightarrow 0_{2}^{+}$and $0_{1}^{+} \rightarrow 0_{5}^{+}$transitions, the isoscalar transition matrix is approximately twice as large as the electric transition matrix reflecting the fact that protons and neutrons equally contribute to these $\alpha$ clustering excitation.

As for the dipole transitions, we confirmed that the both electric and isoscalar dipole transitions are greatly enhanced between the ${ }^{14} \mathrm{C}+\alpha$ cluster states, namely the $1_{4}^{-} \rightarrow 0_{2}^{+}$ and $1_{4}^{-} \rightarrow 0_{5}^{+}$transitions. However, we could not resolve the inconsistency between theories and experiments for the electric dipole transition of the $1_{1}^{-}$state.

\section{ACKNOWLEDGMENT}

The authors acknowledge the support by the grant for the RCNP joint research project at Osaka University and by the collaborative research program 2019 at Hokkaido University. The numerical calculation has been conducted on a supercomputer at Research Center for

Nuclear Physics, Osaka University. One of the author (M.K.) acknowledges the support by the JSPS KAKENHI Grant Number JP19K03859.

[1] A. Arima, H. Horiuchi, and T. Sebe, Physics Letters B 24, 129 (1967).

[2] H. Horiuchi and K. Ikeda, Progress of Theoretical Physics 40, 277 (1968).

[3] B. Buck, C. B. Dover, and J. P. Vary, Physical Review C 11, 1803 (1975).

[4] Y. Suzuki, Progress of Theoretical Physics 55, 1751 (1976).

[5] Y. Suzuki, Progress of Theoretical Physics 56, 111 (1976).

[6] Y. Fujiwara, H. Horiuchi, K. Ikeda, M. Kamimura, K. Kato, Y. Suzuki, and E. Uegaki, Progress of Theoretical Physics Supplement 68, 29 (1980).

[7] P. Descouvemont, D. Baye, and P. H. Heenen, Nuclear Physics, Section A 430, 426 (1984),

[8] A. Cunsolo, A. Foti, G. Immè, G. Pappalardo, G. Raciti, and N. Saunier, Physical Review C 24, 476 (1981).

[9] Y. Alhassid, M. Gai, and G. F. Bertsch, Physical Review Letters 49, 1482 (1982). 
[10] M. Gai, M. Ruscev, A. C. Hayes, J. F. Ennis, R. Keddy, E. C. Schloemer, S. M. Sterbenz, and D. A. Bromley, Physical Review Letters 50, 239 (1983).

[11] M. Gai, R. Keddy, D. A. Bromley, J. W. Olness, and E. K. Warburton, Physical Review C 36, 1256 (1987).

[12] M. Gai, S. L. Rugari, R. H. France, B. J. Lund, Z. Zhao, D. A. Bromley, B. A. Lincoln, W. W. Smith, M. J. Zarcone, and Q. C. Kessel, Physical Review Letters 62, 874 (1989).

[13] N. Curtis, D. D. Caussyn, C. Chandler, M. W. Cooper, N. R. Fletcher, R. W. Laird, and J. Pavan, Physical Review C 66, 024315 (2002).

[14] S. Yildiz, M. Freer, N. Soić, S. Ahmed, N. I. Ashwood, N. M. Clarke, N. Curtis, B. R. Fulton, C. J. Metelko, B. Novatski, N. A. Orr, R. Pitkin, S. Sakuta, and V. A. Ziman, Physical Review C 73, 034601 (2006).

[15] E. D. Johnson, G. V. Rogachev, V. Z. Goldberg, S. Brown, D. Robson, A. M. Crisp, P. D. Cottle, C. Fu, J. Giles, B. W. Green, K. W. Kemper, K. Lee, B. T. Roeder, and R. E. Tribble, The European Physical Journal A 2009 42:2 42, 135 (2009).

[16] W. von Oertzen, T. Dorsch, H. G. Bohlen, R. Krücken, T. Faestermann, R. Hertenberger, T. Kokalova, M. Mahgoub, M. Milin, C. Wheldon, and H. F. Wirth, The European Physical Journal A 2009 43:1 43, 17 (2009).

[17] M. L. Avila, G. V. Rogachev, V. Z. Goldberg, E. D. Johnson, K. W. Kemper, Y. M. Tchuvil'sky, and A. S. Volya, Physical Review C - Nuclear Physics 90, 024327 (2014).

[18] B. Yang, Y. L. Ye, J. Feng, C. J. Lin, H. M. Jia, Z. H. Li, J. L. Lou, Q. T. Li, Y. C. Ge, X. F. Yang, H. Hua, J. Li, H. L. Zang, Q. Liu, W. Jiang, C. G. Li, Y. Liu, Z. Q. Chen, H. Y. Wu, C. G. Wang, W. Liu, X. Wang, J. J. Li, D. W. Luo, Y. Jiang, S. W. Bai, J. Y. Xu, N. R. Ma, L. J. Sun, D. X. Wang, Z. H. Yang, and J. Chen, Physical Review C 99, 064315 (2019).

[19] T. Sakuda, Progress of Theoretical Physics 57, 855 (1977).

[20] T. Sakuda, S. Nagata, and F. Nemoto, Progress of Theoretical Physics 59, 1543 (1978).

[21] H. J. Assenbaum, K. Langanke, and A. Weiguny, Zeitschrift für Physik A: Atoms and Nuclei 318, 35 (1984).

[22] D. Baye and P. Descouvemont, Physics Letters B 146, 285 (1984).

[23] P. Descouvemont and D. Baye, Physical Review C 31, 2274 (1985).

[24] Y. Suzuki, A. Yamamoto, and K. Ikeda, Nuclear Physics, Section A 444, 365 (1985). 
[25] N. Furutachi, M. Kimura, A. Dote, Y. Kanada-En'yo, S. Oryu, A. Doté, Y. Kanada-En'yo, and S. Oryu, Progress of Theoretical Physics 119, 403 (2008).

[26] T. Baba and M. Kimura, Physical Review C 100, 064311 (2019).

[27] F. Ajzenberg-Selove, Nuclear Physics, Section A 392, 1 (1983).

[28] T. Kawabata, H. Akimune, H. Fujita, Y. Fujita, M. Fujiwara, K. Hara, K. Hatanaka, M. Itoh, Y. Kanada-En’yo, S. Kishi, K. Nakanishi, H. Sakaguchi, Y. Shimbara, A. Tamii, S. Terashima, M. Uchida, T. Wakasa, Y. Yasuda, H. Yoshida, and M. Yosoi, Physics Letters B 646, 6 (2007).

[29] Y. Kanada-En'yo, Physical Review C - Nuclear Physics 75, 024302 (2007).

[30] Y. Funaki, T. Yamada, H. Horiuchi, G. Röpke, P. Schuck, and A. Tohsaki, Physical Review Letters 101, 082502 (2008).

[31] T. Yamada, Y. Funaki, H. Horiuchi, K. Ikeda, and A. Tohsaki, Progress of Theoretical Physics 120, 1139 (2008).

[32] M. Ito, Physical Review C - Nuclear Physics 83, 044319 (2011).

[33] T. Ichikawa, N. Itagaki, Y. Kanada-En'Yo, T. Kokalova, and W. Von Oertzen, Physical Review C - Nuclear Physics 86, 031303 (2012).

[34] T. Yamada, Y. Funaki, T. Myo, H. Horiuchi, K. Ikeda, G. Röpke, P. Schuck, and A. Tohsaki, Physical Review C - Nuclear Physics 85, 034315 (2012).

[35] Y. Kanada-En'Yo, Physical Review C - Nuclear Physics 89, 024302 (2014).

[36] Z. H. Yang, Y. L. Ye, Z. H. Li, J. L. Lou, J. S. Wang, D. X. Jiang, Y. C. Ge, Q. T. Li, H. Hua, X. Q. Li, F. R. Xu, J. C. Pei, R. Qiao, H. B. You, H. Wang, Z. Y. Tian, K. A. Li, Y. L. Sun, H. N. Liu, J. Chen, J. Wu, J. Li, W. Jiang, C. Wen, B. Yang, Y. Y. Yang, P. Ma, J. B. Ma, S. L. Jin, J. L. Han, and J. Lee, Physical Review Letters 112, 162501 (2014).

[37] Y. Chiba and M. Kimura, Physical Review C - Nuclear Physics 91, 061302 (2015).

[38] T. Yamada and Y. Funaki, Physical Review C - Nuclear Physics 92, 034326 (2015).

[39] Y. Chiba, M. Kimura, and Y. Taniguchi, Physical Review C 93, 034319 (2016).

[40] Y. Kanada-En'yo, Physical Review C 93, 054307 (2016).

[41] B. Zhou, A. Tohsaki, H. Horiuchi, and Z. Ren, Physical Review C 94, 044319 (2016).

[42] Y. Chiba, Y. Taniguchi, and M. Kimura, Physical Review C 95, 044328 (2017) arXiv:1610.04000.

[43] Y. Kanada-En’Yo and Y. Shikata, Physical Review C 100, 014301 (2019). 
[44] Y. Chiba and M. Kimura, Physical Review C 101, 024317 (2020).

[45] Y. Kanada-En'yo and K. Ogata, Physical Review C 101, 014317 (2020).

[46] J. Berger, M. Girod, and D. Gogny, Computer Physics Communications 63, 365 (1991).

[47] Y. Kanada-En'yo, M. Kimura, and H. Horiuchi, Comptes Rendus Physique 4, 497 (2003).

[48] M. Kimura, Physical Review C 69, 044319 (2004).

[49] Y. Kanada-En'yo, M. Kimura, and A. Ono, Progress of Theoretical and Experimental Physics 2012, 1A202

[50] T. Baba and M. Kimura, Physical Review C 94, 044303 (2016).

[51] T. Baba and M. Kimura, Physical Review C 95 (2017), 10.1103/PhysRevC.95.064318.

[52] D. L. Hill and J. A. Wheeler, Physical Review 89, 1102 (1953).

[53] Y. Chiba and M. Kimura, Progress of Theoretical and Experimental Physics 2017 (2017), 10.1093/ptep/ptx0

[54] N. Itagaki and S. Okabe, Physical Review C 61, 044306 (2000).

[55] W. von Oertzen, M. Freer, and Y. Kanada-En'yo, Physics Reports 432, 43 (2006).

[56] M. Kimura, Physical Review C - Nuclear Physics 75 (2007), 10.1103/PhysRevC.75.034312.

[57] T. Baba, Y. Chiba, and M. Kimura, Physical Review C 90, 064319 (2014).

[58] J. Li, Y. L. Ye, Z. H. Li, C. J. Lin, Q. T. Li, Y. C. Ge, J. L. Lou, Z. Y. Tian, W. Jiang, Z. H. Yang, J. Feng, P. J. Li, J. Chen, Q. Liu, H. L. Zang, B. Yang, Y. Zhang, Z. Q. Chen, Y. Liu, X. H. Sun, J. Ma, H. M. Jia, X. X. Xu, L. Yang, N. R. Ma, and L. J. Sun, Physical Review C 95, 021303 (2017).

[59] K. Ikeda, N. Takigawa, and H. Horiuchi, Progress of Theoretical Physics Supplement E68, 464 (1968). 